\title{
Retinal fluid changes and therapeutic effects in symptomatic circumscribed choroidal hemangioma patients: a long-term follow up study
}

\author{
Junwon Lee ${ }^{1}$, Christopher Seungkyu Lee ${ }^{2}$, Min Kim² $^{2}$ and Sung Chul Lee ${ }^{1^{*}}$ (D)
}

\begin{abstract}
Background: Changes in retinal fluid patterns associated with circumscribed choroidal hemangioma (CCH) have not been investigated yet. A long-term follow-up study was performed to evaluate the changes of retinal fluid patterns and treatment responses.

Methods: We retrospectively reviewed medical records of all CCH patients diagnosed between November 2005 and March 2017. Enrolled patients had visual symptoms, were treatment-naïve, and had been followed-up for more than 2 years. Best corrected visual acuities (BCVA) and the presence, severity, and pattern change of the subretinal fluid (SRF) and intraretinal fluid (IRF) in the macula on optical coherence tomography (OCT) were analyzed at initial presentation and follow-up visits.

Results: Twenty-six patients were enrolled. All patients received one or more of the following treatments: PDT, TT, and intravitreal bevacizumab (Avastin) injection (IVB). Primary therapy consisted of PDT in 9 patients (34.6\%), TTा in 7 patients (26.9\%) and IVB in 10 patients (38.5\%). At initial presentation, the SRF-only pattern was mostly observed. Despite treatment, IRF occurred over time; eventually, advanced cystoid macular oedema (CME) developed. In terms of retinal fluid reduction, PDT was most efficacious $(9 / 9,100 \%)$, and $\Pi T$ and IVB showed moderate efficacy (TाT: 4/7, 57.1\%; IVB: 5/10, 50\%) as a primary therapy. After advanced CME developed, IVB and TाT showed no or minimal effect (TT: 0/1, 0\%; IVB: 0/19, 0\%), and PDT was the only effective therapy (6/10,60\%).

Conclusion: The pattern of retinal fluid accompanied by $\mathrm{CCH}$ evolved from an SRF-only pattern initially to an advanced CME pattern. The effectiveness of treatments decreased over time, and advanced CME generally showed resistance to treatments. PDT would be the most recommended treatment.
\end{abstract}

Keywords: Choroidal hemangioma, Photodynamic therapy, Cystoid macular oedema, Retinal fluid pattern

\section{Background}

Circumscribed choroidal hemangioma $(\mathrm{CCH})$ is a benign tumour, and asymptomatic $\mathrm{CCH}$ does not require treatment. Associated serous retinal detachment and cystoid macular edema (CME) are common findings in symptomatic $\mathrm{CCH}$. Various treatment modalities including photodynamic therapy (PDT) [1-7], transpupillary

\footnotetext{
* Correspondence: sunglee@yuhs.ac

${ }^{1}$ Department of Ophthalmology, Eye and ENT Hospital, Severance Hospital, Institute of Vision Research, Yonsei University College of Medicine, 50-1 Yonsei-ro, Seodaemun-gu, Seoul 03722, South Korea

Full list of author information is available at the end of the article
}

thermotherapy (TTT) [8-10], anti-vascular endothelial growth factor (VEGF) injections [8], lens-sparing external beam radiotherapy $[11,12]$, plaque brachytherapy $[13,14]$, proton beam therapy [15], stereotactic radiosurgery [16], laser photocoagulation [11], and oral propranolol [17] have been applied for treating $\mathrm{CCH}$ related symptomatic fluids. Although various treatments have been explored, according to recent studies, PDT has emerged as the treatment of choice with high rates of tumour regression, fluid resorption and minimal complications $[4-7,18]$.

In a large study, serous retinal detachment and CME were reported in 81 and $17 \%$ of patients at initial

(c) The Author(s). 2018 Open Access This article is distributed under the terms of the Creative Commons Attribution 4.0 International License (http://creativecommons.org/licenses/by/4.0/), which permits unrestricted use, distribution, and reproduction in any medium, provided you give appropriate credit to the original author(s) and the source, provide a link to the Creative Commons license, and indicate if changes were made. The Creative Commons Public Domain Dedication waiver (http://creativecommons.org/publicdomain/zero/1.0/) applies to the data made available in this article, unless otherwise stated. 
presentation, respectively [19]. However, there have been no longitudinal observational studies to investigate changes in retinal fluid patterns associated with $\mathrm{CCH}$.

The aim of this study was to evaluate the changes in retinal fluid patterns, the response to various treatment modalities, and the prognosis of visual acuity in patients with $\mathrm{CCH}$ through a long-term follow-up.

\section{Methods}

We retrospectively reviewed the medical records of all patients diagnosed with $\mathrm{CCH}$ at Yonsei University Severance Hospital between November 2005 and March 2017. Patients who had visual symptoms, were treatment-naïve at initial presentation, and were followed-up for more than 2 years were enrolled. $\mathrm{CCH}$ was diagnosed based on fundus examination, indocyanine green angiography (ICGA), and ultrasonography. This study was approved by the Institutional Review Board at Yonsei University Medical Center (Reference No. 4-2017-0955) and adhered to the tenets of the Declaration of Helsinki.

Baseline demographic data, including age, sex, general and ocular history, symptoms, follow-up duration, and series of treatments were recorded.

Initial assessment of patients included the minimal angle of resolution (logMAR) best-corrected visual acuity (BCVA) measurement using the Snellen visual acuity chart, slit-lamp biomicroscopy, dilated fundus examination, fundus photography, fluorescein angiography and ICGA, B-scan ultrasonography, and optical coherence tomography (OCT) (Stratus III OCT, Carl Zeiss, Dublin, California, USA; or Spectralis HRA + OCT, Heidelberg Engineering, Heidelberg, Germany).

Tumour size and location, and distance to the foveola and optic disc margin were measured using B-scan ultrasonography, fundus photography, and OCT.

$\mathrm{BCVA}$, and the presence, severity, and pattern change of the retinal fluid on OCT were observed during every follow-up visit. Changes in retinal fluid following each treatment were evaluated qualitatively and quantitatively.

The pattern of retinal fluid in the macula was divided into three types; subretinal fluid (SRF) only; SRF / intraretinal fluid (IRF) combined; and advanced (well organized) CME pattern consisting of severe IRF.

\section{Treatment modalities}

"All patients received one or more of the following treatments: PDT, TTT, and intravitreal bevacizumab (Avastin) injection (IVB)."

PDT was performed with standard equipment under standard conditions. An 83-s laser spot at $689 \mathrm{~nm} \mathrm{(50} \mathrm{J/}$ $\mathrm{cm}^{2}$ ) coupled with intravenous verteporfin was used to treat the entire $\mathrm{CCH}$. Verteporfin 6 (typical) or $12 \mathrm{mg} /$ $\mathrm{m}^{2}$ (enhanced) was injected. The area of treatment was determined by the size of the tumour measured using
ICGA. When the target area exceeded the maximum spot size, treatments were repeated several times without overlap.

TTT was performed under topical anesthesia via a dilated pupil. Patients were treated with an infrared diode laser at $810 \mathrm{~nm}$ using a slit-lamp biomicroscope delivery system. Each tumour was covered entirely with confluent laser spots, with the power ranging from 200 to 500 $\mathrm{mW}$ and spot size between 1200 and $3000 \mu \mathrm{m}$ to induce a slight color change with $1 \mathrm{~min}$ of exposure at each spot. An area of 1-disc diameter (DD) around the foveola and $1 \mathrm{DD}$ around the disc margin were spared during TTT in all cases.

Using an aseptic technique, bevacizumab (Avastin) $1.25 \mathrm{mg}$ was injected 3.0 or $3.5 \mathrm{~mm}$ posterior to the limbus through the pars plana using a 30-gauge needle in the operating room.

\section{Comprehensive evaluation of therapeutic response}

Indications for treatment included serous macular detachment (SMD) and CME causing visual symptoms. When the fluid was fully resolved, no further treatment was performed. When the treatment effect was insufficient or the fluid recurred, treatment was performed again. When the treatment appeared to be ineffective or an additional session using the same treatment modality had the potential to induce retinal damage, a different therapy was trialled. OCT was performed within 8 weeks after each treatment. The treatment effect was evaluated after 4 weeks in cases where IVB was administered. In cases where patients received PDT or TTT treatment, monitoring continued for at least 8 weeks.

During long-term follow-up, the types, order, and number of treatments administered varied among patients. For analysis, the series of treatments were simplified. The efficacy of each treatment was assessed by whether it increased or decreased the amount of SRF and/or IRF on OCT. Efficacy was classified into three groups and was numerically parameterized.

A reduction of less than $20 \%$, or no change, in the fluid following treatment was classified as 'minimal or none' and parameterized as 0 point. For cases with a 20 to $80 \%$ reduction following treatment, these were classified as 'partial' responses and parameterized as 0.5 point. For cases with an 80 to $100 \%$ reduction, these were classified as 'almost or complete' responses and parameterized as 1 point. The mean score for several sessions of a single treatment modality was obtained when the same treatment modality was applied continuously in a series.

For example, if IVBs were performed 5 times consecutively and the effects were \#1: almost or complete; \#2: partial; \#3: minimal or none; \#4: minimal or none; and \#5: partial, the overall mean efficacy of IVB was 0.4 (1.0 $+0.5+0+0+0.5 / 5=2.0 / 5$ ) . 


\section{Statistics}

Statistical analyses were performed using SPSS 23.0 software (IBM Corp., Armonk, NY, USA). Averages are reported as the mean \pm standard deviation (SD). When comparing the paired mean at different points within an individual, a paired t-test was performed. A $p$-value of $<$ 0.05 was considered statistically significant.

\section{Results}

All enrolled patients had symptomatic $\mathrm{CCH}$ and were treatment-naïve. All patients received PDT and/or TTT and/or IVB for serous macular detachment and CME. Patient demographics and clinical data are shown in Table 1.

Twenty-six patients were enrolled. The mean \pm SD (minimum to maximum) follow-up duration was 63.68 \pm 30.10 (range, 27.77 to 128.52 ) months. The median and interquartile ranges of follow-up duration were 58.33 months and 35.73 to 82.02 months, respectively. Seventeen patients were followed-up for 48 months and 9 patients were followed up for 24 to 48 months.

The mean age was $49.26 \pm 10.07$ years. There were 16 males and 10 females. The mean largest base diameter (LBD) and height of the tumour were $8.70 \pm 1.73$ (range, 4.56-11.86) and $3.31 \pm 1.02$ (range, 1.52-5.21) $\mathrm{mm}$, respectively. Twelve tumours involved the subfoveal area and 14 tumours were located in the extrafoveal area.

\section{Pattern change of retinal fluid related with $\mathrm{CCH}$}

The pattern changes in retinal fluid associated with $\mathrm{CCH}$ are presented in Fig. 1. The pattern of retinal fluid at initial presentation was SRF-only in 19 patients (73.1\%), SRF and IRF combined in 4 patients (15.4\%), and advanced CME in 3 patients (11.5\%).

Over time, the pattern of retinal fluid evolved. Among the patients with more than 48 months' follow-up, the retinal fluid pattern progressed to an advanced CME pattern in 9 of 17 patients (52.9\%), changed to SRF and IRF combined pattern in 6 patients (35.3\%), and remained as SRF-only in only 2 patients (11.8\%). IRF occurred in 2 out of 9 (22.2\%) patients with 24 to 48 months' follow-up.

Overall, the SRF-only pattern was observed mostly at initial presentation, whereas IRF occurred over time. The advanced CME pattern was observed mostly with patients who had long-term follow-up.

There was no association between the use of certain treatments and the development of advanced CME.

\section{Therapeutic effect on SRF or IRF according to treatment modalities}

We examined the therapeutic response of retinal fluid according to each treatment modality performed as a primary therapy or secondary therapy.

Primary therapy was PDT in 9 patients (typical 7; enhanced 2), TTT in 7 patients and IVB in 10 patients. For
PDT cases, 'almost or complete' response (mean score 1.0) was observed in 8 of 9 patients (typical 6; enhanced 2) $(88.9 \%)$ and a 'partial' response (mean score 0.5 ) was observed in 1 patient (11.1\%). For TTT cases, 3 patients (42.9\%) showed 'almost or complete' resolution (mean score 1.0), 1 (14.3\%) showed 'partial' resolution (mean score 0.5 ) and $3(42.9 \%)$ showed 'minimal or no' effect (mean score 0.0). In IVB cases, 2 patients (20\%) showed 'partial to almost' resolution (mean score 0.75), 3 patients showed 'partial' response (mean score 0.5) (30\%) and 5 patients (50\%) showed 'minimal or no' effect (mean score 0.0). PDT had a good therapeutic effect, and TTT and IVB showed modest therapeutic effects on retinal fluid with $\mathrm{CCH}$.

Secondary therapy was performed as follows: PDT in 10 patients (typical 5; enhanced 5), TTT in 1 patient and IVB in 7 patients. TTT and IVB showed 'minimal or no' effect (mean score 0.0) in all patients. PDT showed 'almost or complete' resolution (mean score 1.0) in 5 patients (typical 2; enhanced 3) (50\%), 'partial to almost' resolution (mean score 0.75 ) in 2 patients (typical 2) (20\%) and 'partial' response (mean score 0.5) in 3 patients (typical 1; enhanced 2) (30\%). Typical PDT and enhanced PDT showed similar effects. For all 3 treatment modalities, there was a lower therapeutic effect when they were applied as a secondary therapy compared to when they were used as a primary therapy.

Nine patients showed advanced CME during the follow-up period. IVBs were performed 19 times in 6 patients and showed 'minimal or no' effect (mean score 0.0) in all cases. TTT was performed once in 1 patient and showed 'minimal or no' effect (mean score 0.0). PDTs were performed 10 times (typical 6 times; enhanced 4 times) in 5 patients. Four out of 10 (40\%) sessions showed 'almost or complete' response (mean score 1.0) and 2 out of 10 (20\%) showed a 'partial' response (mean score 0.5), and 4 out of 10 (40\%) showed 'no or minimal' effect (mean score 0.0). Enhanced PDT was not superior to typical PDT. IVB and TTT had no effect on advanced CME.

\section{Therapeutic effect on BCVA according to treatment modalities}

Results from final BCVA compared with initial BCVA according to primary and secondary therapeutic modalities are presented in Table 2. Final BCVA improved in 9 out of 26 patients $(34.6 \%)$, remained stable in 4 patients (15.4\%), and deteriorated in 13 patients (50\%) compared to initial BCVA. The mean final BCVA was lower than the initial BCVA with marginal significance [logMAR (Initial vs. Final): $0.79 \pm 0.54$ vs. $1.10 \pm 0.91(p=0.064)$ ]

We further examined BCVA changes according to each treatment modality. Among 17 patients followed up over 48 months, as primary and secondary therapies, 8 patients received PDT at least once and 8 patients 


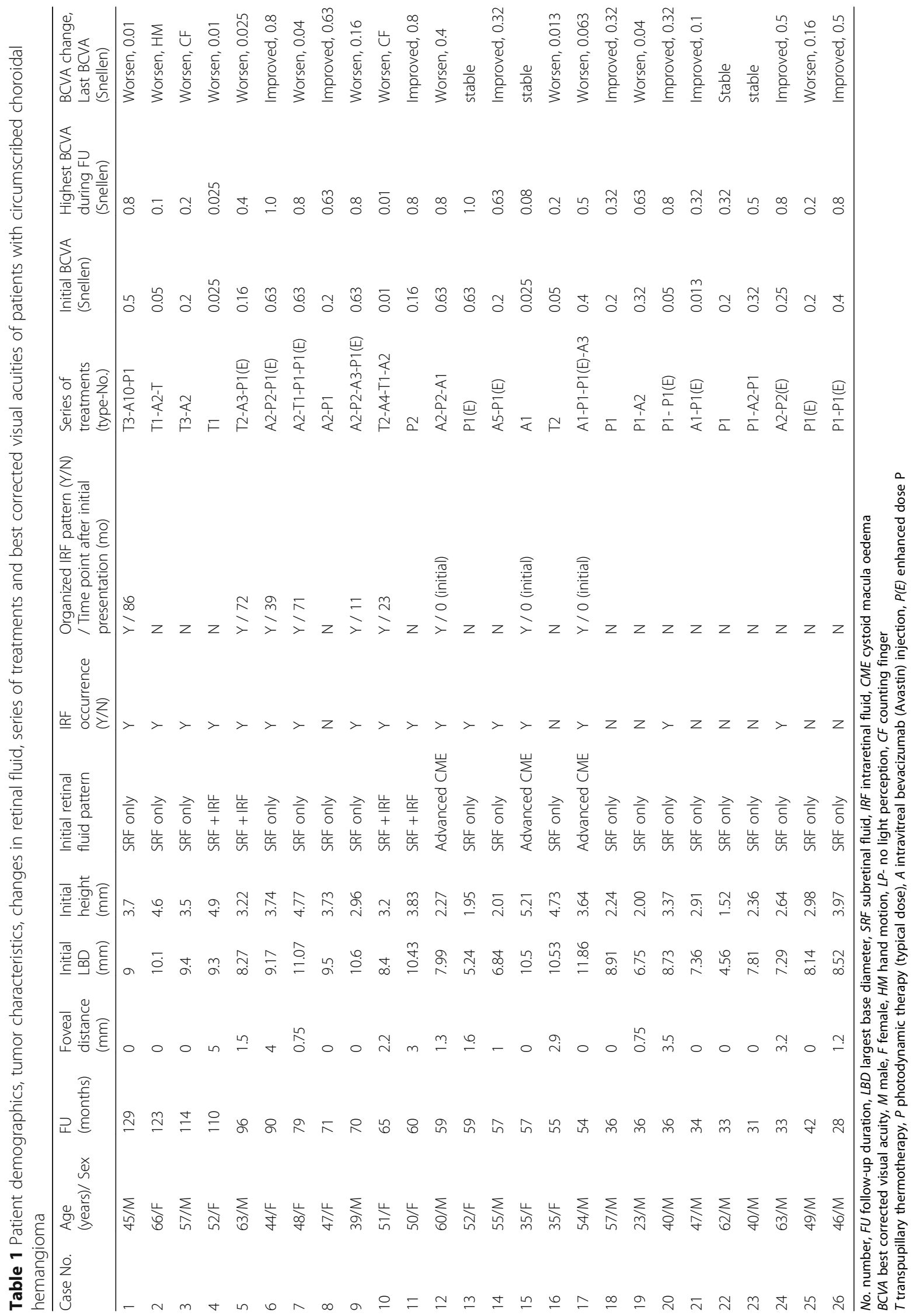




\section{Retinal fluid pattern}

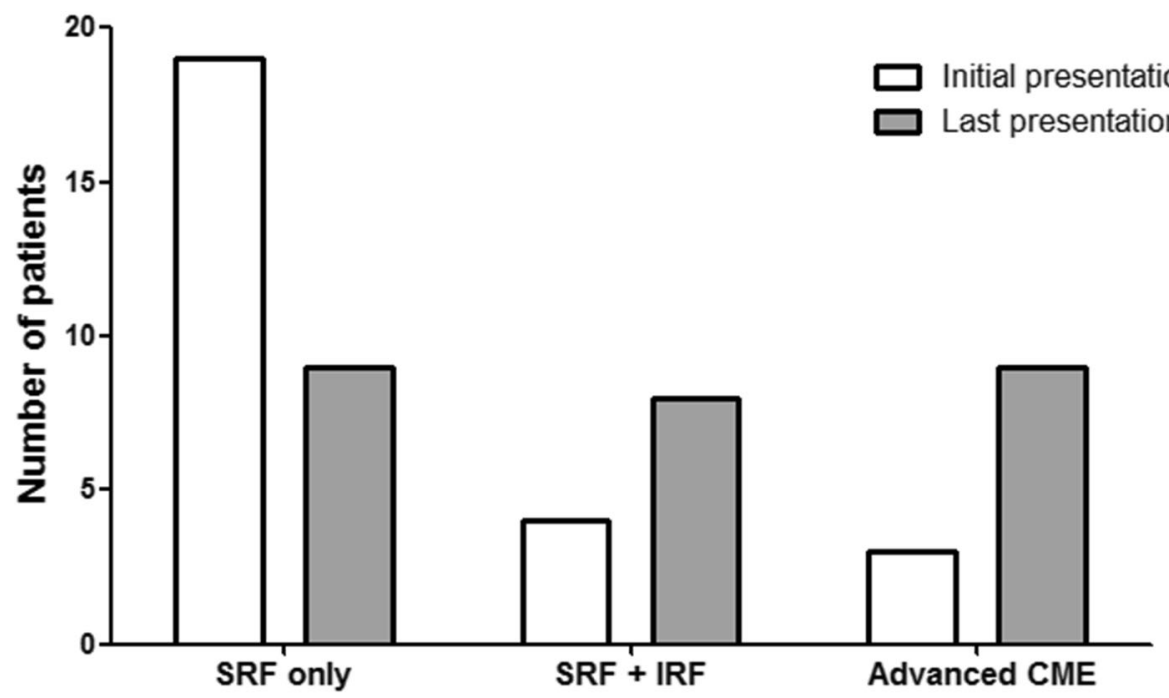

Fig. 1 Changes in retinal fluid patterns over time in patients with circumscribed choroidal hemangioma. Among enrolled patients, patterns of retinal fluid at initial presentation were as follows: SRF-only in 19 patients (19/26, 73.1\%), SRF and IRF combined in 4 patients (4/26, 15.4\%), and advanced cystoid macular edema (CME) in 3 patients (3/26, 11.5\%). Over time, changes in the pattern of retinal fluid occurred. The patterns of retinal fluid at last presentation were as follows: Advanced CME in 9 of 26 patients $(9 / 26,34.6 \%)$, SRF and IRF combined in 8 patients (8/26, $30.8 \%)$, and remained as SRF-only in 9 patients $(9 / 26,34.6 \%)$

received TTT at least once. There was no significant difference in age, tumour position, and tumour size between the PDT group and the TTT group. Comparing final BCVA with initial BCVA, in PDT cases, 4 out of 8 patients (50\%) showed an improvement in BCVA, 1 patient (12.5\%) remained stable and 3 patients $(37.5 \%)$ deteriorated. Whereas, all patients that received TTT showed deterioration in their BCVA (8/ $8=100 \%)$.

All 9 patients who were followed up for 24 to 48 months received PDT plus IVB (or IVB plus PDT). Comparing final BCVA with initial BCVA, 5 out of 9 patients (55.6\%) showed improvement in their BCVA, 2 patients $(22.2 \%)$ remained stable, and 2 patients' $(22.2 \%)$ BCVA deteriorated. The mean final BCVA was improved when compared to the initial BCVA, however, this was not statistically significant $(p=0.473)$.

\section{Cases}

We present two representative cases demonstrating the pattern of change in retinal fluid associated with $\mathrm{CCH}$. In both cases, SRF-only patterns were noted at initial presentation and over time, SRF transitioned to IRF, eventually progressing to advanced CME, despite various treatment efforts. In one case (Fig. 2; Case 7), the retinal fluid responded well to PDT initially, however, the fluid eventually returned and subsequently progressed to the advanced CME pattern. In the other case (Fig. 3; Case 6), the retinal fluid was completely resolved following multiple treatments with PDT, and there was no recurrence during the more than 3 years of follow-up.

\section{Discussion}

We investigated the changes in retinal fluid patterns and the therapeutic response to various treatment modalities

Table 2 Final best corrected visual acuity (BCVA) compared with initial BCVA according to primary and secondary therapeutic modalities

\begin{tabular}{|c|c|c|c|c|}
\hline & BCVA change & & & \\
\hline Treatment modality & Improved & Stable & Worsen & Total number \\
\hline$P+A(A+P)$ & $5(3+2)(45.45 \%)$ & $1(0+1)(9.09 \%)$ & $5(3+2)(45.45 \%)$ & 11 \\
\hline$P$ & $4(1+3)(66.67 \%)$ & $2(1+1)(33.33 \%)$ & & 6 \\
\hline A & & $1(1+0)(100 \%)$ & & 1 \\
\hline$T+A(A+T)$ & & & $6(6+0)(100 \%)$ & 6 \\
\hline $\mathrm{T}$ & & & $2(2+0)(100 \%)$ & 2 \\
\hline
\end{tabular}

Total number of patients (number of patients followed up over 48 months + number of patients followed up between 24 to 48 months) $B C V A$ best corrected visual acuity, $T$ transpupillary thermotherapy, $P$ photodynamic therapy, $A$ intravitreal bevacizumab (Avastin) injection 


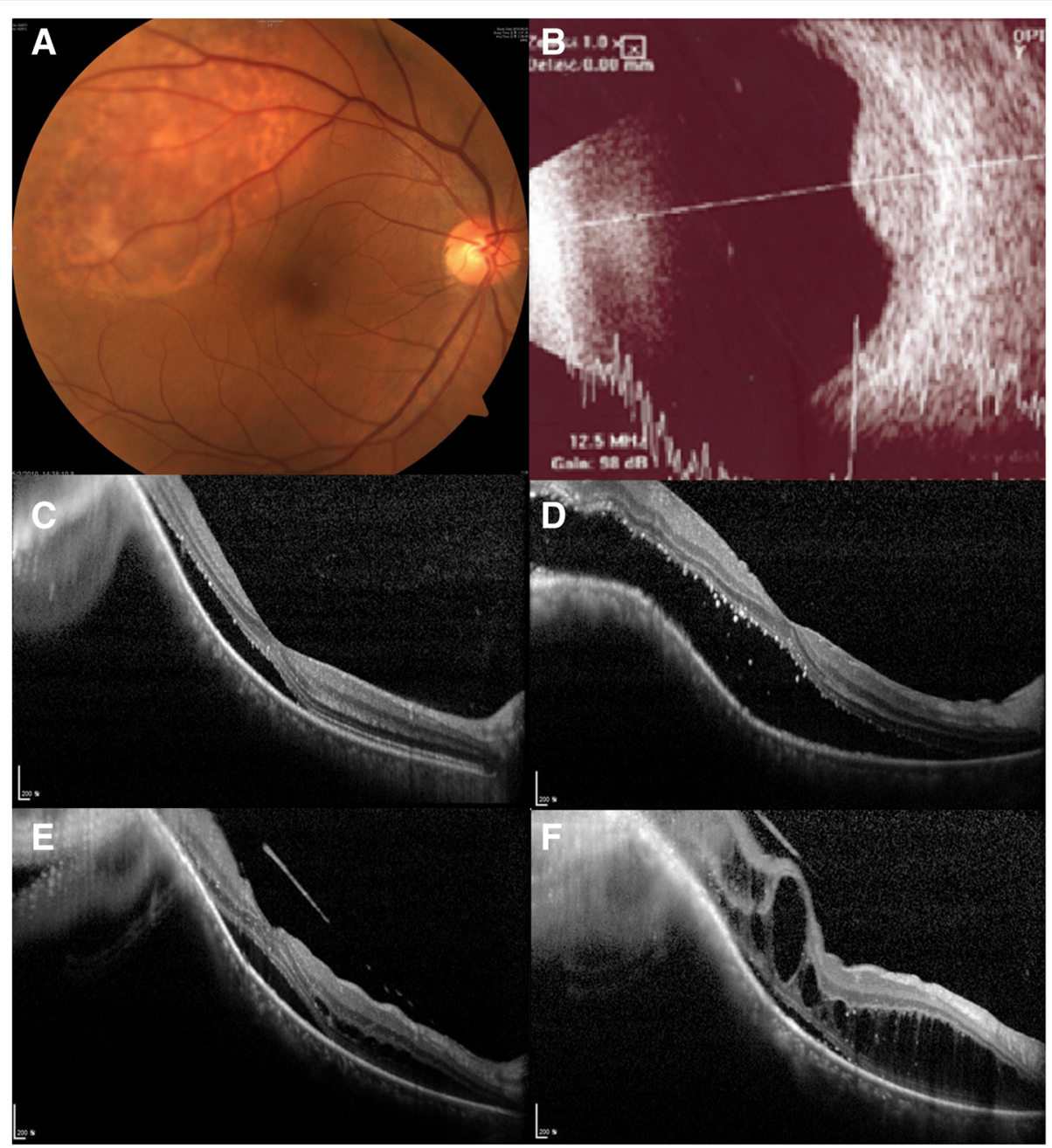

Fig. 2 A representative case of circumscribed choroidal hemangioma showing the change in retinal fluid over time. A 48-year-old woman (Case 7) presented with blurred vision of the right eye. Her initial best corrected visual acuity (BCVA) was 0.63. (a) On fundus photography, an orange and red-colored subretinal mass was observed supero-temporally to the macula. (b) B-scan ultrasonography showed a hyperreflective mass with a height of $4.77 \mathrm{~mm}$ and base diameter of $11.07 \mathrm{~mm}$. (c) Shallow subretinal fluid involving the fovea was observed along with elevation of the choroid by a mass lesion on optical coherence tomography. (d) During the first 6 months, despite a single intravitreal bevacizumab (IVB) injection, the subretinal fluid (SRF) increased. Thereafter, a second IVB injection and single session of transpupillary thermotherapy (TT) were performed; however, there was no significant improvement in therapeutic effect. Subsequently, a single session of photodynamic therapy (PDT) and enhanced PDT were performed and the SRF completely resolved. However, the subsequent BCVA was 0.04. (e) After 2 years of follow-up, SRF and intraretinal fluid then developed. (f) Six months later, an advanced cystoid macular edema pattern was observed

in patients with symptomatic $\mathrm{CCH}$ who underwent long-term follow-up. There have been no studies that focus on changes in retinal fluid patterns associated with $\mathrm{CCH}$.

The SRF-pattern was mainly observed in the early stage, followed by SRF and IRF combined, and eventually, an advanced CME pattern was established over time. During the follow-up period, advanced CME occurred regardless of the treatment modality. Despite treatment efforts, the occurrence of CME could not be prevented. Patients with advanced CME at initial presentation may be assumed to have had long-lasting retinal fluid along with $\mathrm{CCH}$.
The pathophysiology of CME in $\mathrm{CCH}$ is poorly understood. CME often occurs due to breakdown of the inner blood-retina barrier and is not an uncommon manifestation of diabetic retinopathy, retinal vein occlusion, and inflammatory diseases of the posterior segment. Less commonly, CME is the result of incompetence of the outer blood-retina barrier [20]. In long-standing central serous chorioretinopathy, some eyes will develop CME $[21,22]$. In the case of CME following a long lasting SRF, it may be related to alteration of the external limiting membrane (ELM), which is the linear aggregate of junctions between the outer portions of Müller cells and the inner segments of the photoreceptors. The ELM 


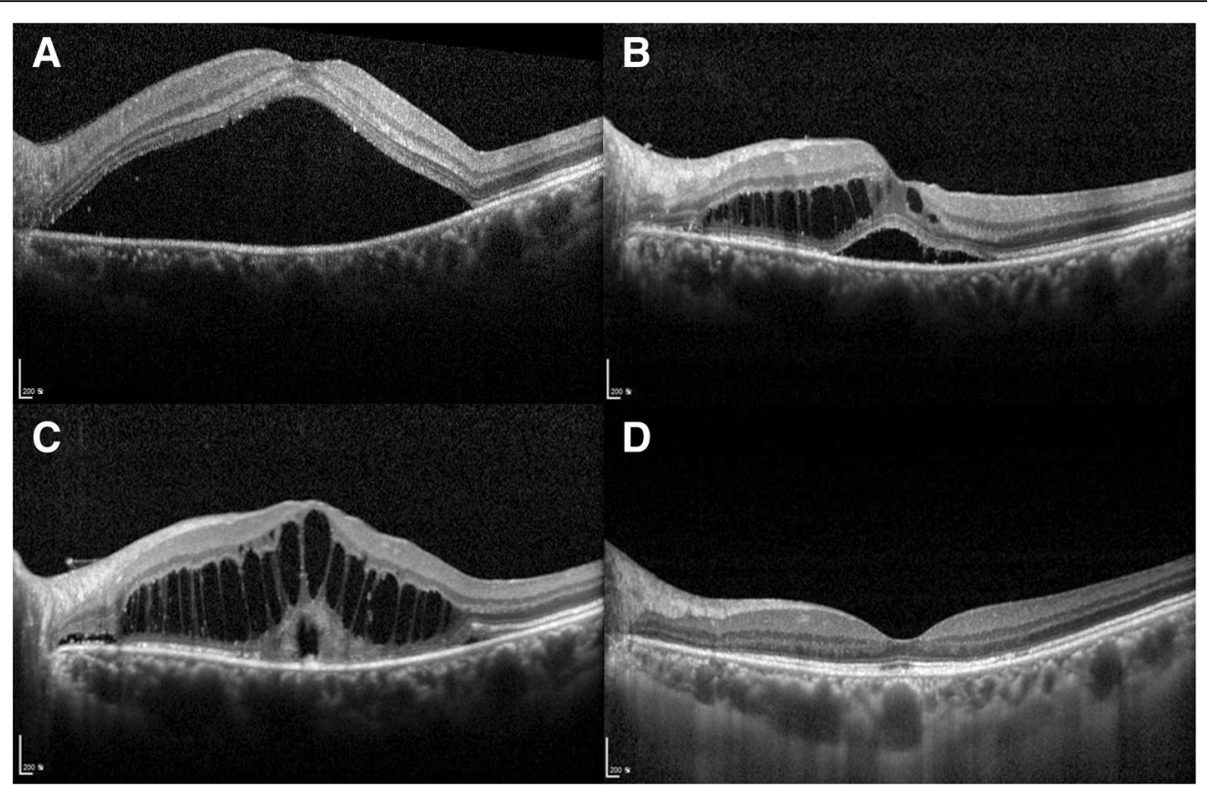

Fig. 3 A representative case of circumscribed choroidal hemangioma where complete resolution of advanced cystoid macular edema (CME) occurred using photodynamic therapy (PDT). A 44-year-old woman (Case 6) presented with blurred vision of the left eye. Her initial best corrected visual acuity (BCVA) was 0.63. At initial presentation, minimal subretinal fluid (SRF) was present. (a) After 8 months, the BCVA decreased to 0.025 , and a large serous retinal detachment (SRD) developed. A single intravitreal bevacizumab injection was performed, and the SRD completely resolved. (b) After 28 months, SRF and intraretinal fluid (IRF) occurred. A second intravitreal bevacizumab injection was performed; however, the fluid only partially resolved. (c) Three months later, advanced CME developed. Two subsequent PDTs were performed, and complete resolution occurred. Thereafter, there was a minor recurrence of SRF and IRF, and enhanced PDT was performed, and the fluid was again completely resolved. (d) After more than 3 years of follow-up, the fluid did not recur

may serve as a barrier for fluid leaving the retina to be pumped from the subretinal space by the retinal pigment epithelium. When the ELM is intact, fluid from below the retina can cause serous detachment of the retina, and when the ELM is defective, there may be passage of fluid into the outer retina [23].

Among the three treatment modalities reported in this study, PDT showed the greatest treatment efficacy, and TTT and IVB showed moderate efficacy for retinal fluid reduction as a primary therapy. As secondary therapies, the efficacy of all 3 treatment modalities was reduced. As the retinal fluid with the tumour became older or recurred, it appears to have become refractory to various treatments. Early and appropriate treatment of $\mathrm{CCH}$ is important.

Several years ago, our group reported the efficacy of IVB in the treatment of 12 symptomatic $\mathrm{CCH}$ patients [8]. IVB led to the resolution of serous macular detachment and improvement of visual acuity in 5 of 9 patients. However, its duration of treatment effectiveness seemed to be relatively short. Similar results were observed in this study. Although IVB may have the advantages of greater feasibility and lower cost than PDT or TTT, IVB monotherapy seems to be insufficiently effective to manage $\mathrm{CCH}$ completely. Repeated IVB therapy should not delay PDT therapy.

Our study findings may have clinical significance because different therapeutic responses were observed depending on the retinal fluid pattern. CME has been considered a poor prognostic factor in $\mathrm{CCH}[2,24]$. A previous study by our group showed that CME does not respond effectively to IVB [8]. In this study, after formation of advanced CME, IVB was not effective in all 19 applications, and PDT was effective in half of the cases. The Shields group also previously reported a case where PDT was effective in CME with $\mathrm{CCH}$ [24].

It is not clear whether the treatment response to advanced CME is poor due to inherent characteristics of the $\mathrm{CME}$ or as a result of long-standing $\mathrm{CCH}$. Alternatively, the poor response may be due to the long-term presence of retinal fluid or damage to the structure of the retinal by previous treatments. Each of these reasons alone or in combination may explain this observation.

According to recent research in $\mathrm{CCH}$ treatment, PDT has emerged as the treatment of choice with high rates of tumour regression, fluid resorption, and minimal complications $[4-7,18]$. Our findings agree with this research as PDT showed the most promising results not only as a primary therapy, but also after the formation of advanced CME.

\section{Conclusion}

Among patients with long-term follow-up, over half of the patients treated with PDT showed improvement in their BCVAs. In conclusion, PDT appears to be an effective treatment for symptomatic retinal fluid associated with $\mathrm{CCH}$. 


\section{Abbreviations}

BCVA: Best corrected visual acuities; CCH: circumscribed choroidal hemangioma; CME: cystoid macular edema; DD: disc diameter; ELM: external limiting membrane; ICGA: indocyanine green angiography; IRF: intraretinal fluid; IVB: intravitreal bevacizumab injection; LBD: largest base diameter; LogMAR: log of the minimum angle of resolution; OCT: optical coherence tomography; PDT: photodynamic therapy; SMD: serous macular detachment; SRF: subretinal fluid; TT: transpupillary thermotherapy; VEGF: vascular endothelial growth factor

\section{Acknowledgements}

None.

\section{Funding}

This research was supported by grant of the Basic Science Research Program through the National Research Foundation of Korea (NRF) funded by the Korea government (Ministry of Science and ICT) (grant number:

2018R1C1B6002732). The funding body had no role in the design or conduct of this research.

\section{Availability of data and materials}

The datasets used and/or analyzed during the current study available from the corresponding author on reasonable request.

\section{Authors' contributions}

Conceptualization of the study: JL and SCL. Data acquisition and analysis: JL, CSL, MK. Manuscript preparation: JL and SCL. All authors have read and approved the manuscript for publication.

\section{Ethics approval and consent participate}

This study was approved by the Institutional Review Board of Severance hospital (IRB No. 4-2017-0955) and was conducted in accordance with the Declaration of Helsinki. Informed consent was obtained from each participant.

\section{Consent for publication}

Consent for publication was obtained from all enrolled patients.

\section{Competing interests}

The authors declare that they have no competing interests.

\section{Publisher's Note}

Springer Nature remains neutral with regard to jurisdictional claims in published maps and institutional affiliations.

\section{Author details}

'Department of Ophthalmology, Eye and ENT Hospital, Severance Hospital, Institute of Vision Research, Yonsei University College of Medicine, 50-1 Yonsei-ro, Seodaemun-gu, Seoul 03722, South Korea. ${ }^{2}$ Department of Ophthalmology, Institute of Human Barrier Research, Gangnam Severance Hospital, Yonsei University College of Medicine, Seoul, South Korea.

\section{Received: 21 July 2018 Accepted: 4 December 2018}

\section{Published online: 13 December 2018}

\section{References}

1. Jurklies B, Anastassiou G, Ortmans S, Schuler A, Schilling H, Schmidt-Erfurth U, Bornfeld N. Photodynamic therapy using verteporfin in circumscribed choroidal haemangioma. Br J Ophthalmol. 2003;87(1):84-9.

2. Porrini G, Giovannini A, Amato G, loni A, Pantanetti M. Photodynamic therapy of circumscribed choroidal hemangioma. Ophthalmology. 2003;110(4):674-80.

3. Singh AD, Kaiser PK, Sears JE, Gupta M, Rundle PA, Rennie IG. Photodynamic therapy of circumscribed choroidal haemangioma. Br J Ophthalmol. 2004; 88(11):1414-8

4. Blasi MA, Tiberti AC, Scupola A, Balestrazzi A, Colangelo E, Valente P, Balestrazzi E. Photodynamic therapy with verteporfin for symptomatic circumscribed choroidal hemangioma: five-year outcomes. Ophthalmology. 2010;117(8):1630-7.

5. Zhang Y, Liu W, Fang Y, Qian J, Xu G, Wang W, Li L, Shen Y, Gao Q. Photodynamic therapy for symptomatic circumscribed macular choroidal hemangioma in Chinese patients. Am J Ophthalmol. 2010;150(5):710-5 e711.
6. Tsipursky MS, Golchet PR, Jampol LM. Photodynamic therapy of choroidal hemangioma in sturge-weber syndrome, with a review of treatments for diffuse and circumscribed choroidal hemangiomas. Surv Ophthalmol. 2011; 56(1):68-85.

7. Jurklies B, Bornfeld N. The role of photodynamic therapy in the treatment of symptomatic choroidal hemangioma. Graefes Arch Clin Exp Ophthalmol. 2005;243(5):393-6.

8. Kwon HJ, Kim M, Lee CS, Lee SC. Treatment of serous macular detachment associated with circumscribed choroidal hemangioma. Am J Ophthalmol. 2012;154(1):137-45 e131.

9. García Arumí J, Ramsay LS, Guraya BC. Transpupillary thermotherapy for circumscribed choroidal hemangiomas. Ophthalmology. 2000;107(2):351-6 discussion 357.

10. Gunduz K. Transpupillary thermotherapy in the management of circumscribed choroidal hemangioma. Surv Ophthalmol. 2004;49(3):316-27.

11. Madreperla SA, Hungerford JL, Plowman PN, Laganowski HC, Gregory PT. Choroidal hemangiomas: visual and anatomic results of treatment by photocoagulation or radiation therapy. Ophthalmology. 1997;104(11):1773-8 discussion 1779

12. Schilling H, Sauerwein W, Lommatzsch A, Friedrichs W, Brylak S, Bornfeld N, Wessing A. Long-term results after low dose ocular irradiation for choroidal haemangiomas. Br J Ophthalmol. 1997;81(4):267-73.

13. Lopez-Caballero C, Saornil MA, De Frutos J, Bianciotto C, Muinos Y, Almaraz A, Lopez-Lara F, Contreras I. High-dose iodine-125 episcleral brachytherapy for circumscribed choroidal haemangioma. Br J Ophthalmol. 2010;94(4):470-3.

14. Naseripour M, Maleki A, Astaraki A, Sedaghat A, Jaberi R, Lee S, Azma Z, Silpa-Archa S. Ruthenium-106 brachytherapy in the treatment of circumscribed choroidal hemangioma. Retina. 2017.

15. Levy-Gabriel C, Rouic LL, Plancher C, Dendale R, Delacroix S, Asselain B, Habrand JL, Desjardins L. Long-term results of low-dose proton beam therapy for circumscribed choroidal hemangiomas. Retina. 2009;29(2):170-5.

16. Song WK, Byeon SH, Kim SS, Kwon OW, Lee SC. Gamma knife radiosurgery for choroidal haemangiomas with extensive exudative retinal detachment. Br J Ophthalmol. 2009;93(6):836-7.

17. Tanabe H, Sahashi K, Kitano T, Tomita Y, Saito AM, Hirose H. Effects of oral propranolol on circumscribed choroidal hemangioma: a pilot study. JAMA Ophthalmol. 2013;131(12):1617-22.

18. Karimi S, Nourinia R, Mashayekhi A. Circumscribed choroidal hemangioma. J Ophthalmic Vis Res. 2015;10(3):320-8.

19. Shields CL, Honavar SG, Shields JA, Cater J, Demirci H. Circumscribed choroidal hemangioma: clinical manifestations and factors predictive of visual outcome in 200 consecutive cases. Ophthalmology. 2001;108(12): 2237-48.

20. Hajali M, Fishman GA, Anderson RJ. The prevalence of cystoid macular oedema in retinitis pigmentosa patients determined by optical coherence tomography. Br J Ophthalmol. 2008;92(8):1065-8.

21. Yannuzzi LA, Shakin JL, Fisher YL, Altomonte MA. Peripheral retinal detachments and retinal pigment epithelial atrophic tracts secondary to central serous pigment epitheliopathy. Ophthalmology. 1984;91(12):1554-72.

22. Schatz H, Osterloh MD, McDonald HR, Johnson RN. Development of retinal vascular leakage and cystoid macular oedema secondary to central serous chorioretinopathy. Br J Ophthalmol. 1993;77(11):744-6.

23. Spaide RF. RETINAL VASCULAR CYSTOID MACULAR EDEMA: review and new theory. Retina. 2016;36(10):1823-42.

24. Shields CL, Materin MA, Marr BP, Mashayekhi A, Shields JA. Resolution of advanced cystoid macular edema following photodynamic therapy for choroidal hemangioma. Ophthalmic Surg Lasers Imaging. 2005;36(3):237-9.

Ready to submit your research? Choose BMC and benefit from:

- fast, convenient online submission

- thorough peer review by experienced researchers in your field

- rapid publication on acceptance

- support for research data, including large and complex data types

- gold Open Access which fosters wider collaboration and increased citations

- maximum visibility for your research: over $100 \mathrm{M}$ website views per year

At $\mathrm{BMC}$, research is always in progress.

Learn more biomedcentral.com/submission 